\title{
Electromagnetic Mathematical Modeling of 3D Supershaped Dielectric Lens Antennas
}

\author{
L. Mescia, ${ }^{1}$ P. Bia, ${ }^{2}$ D. Caratelli, ${ }^{3,4}$ M. A. Chiapperino, ${ }^{1}$ O. Stukach, ${ }^{4}$ and J. Gielis ${ }^{5}$ \\ ${ }^{1}$ Dipartimento di Ingegneria Elettrica e dell'Informazione, Politecnico di Bari, Via Edoardo Orabona 4, 70125 Bari, Italy \\ ${ }^{2}$ EmTeSys S.R.L, 70122 Bari, Italy \\ ${ }^{3}$ The Antenna Company Nederland B.V., High Tech Campus, 5656 AE Eindhoven, Netherlands \\ ${ }^{4}$ Institute of Cybernetics, Tomsk Polytechnic University, 84/3 Sovetskaya Street, Tomsk 634050, Russia \\ ${ }^{5}$ University of Antwerp, Groenenborgerlaan 171, 2020 Antwerp, Belgium
}

Correspondence should be addressed to L. Mescia; luciano.mescia@poliba.it

Received 1 October 2015; Revised 14 January 2016; Accepted 7 February 2016

Academic Editor: Thierry Floquet

Copyright (C) 2016 L. Mescia et al. This is an open access article distributed under the Creative Commons Attribution License, which permits unrestricted use, distribution, and reproduction in any medium, provided the original work is properly cited.

The electromagnetic analysis of a special class of 3D dielectric lens antennas is described in detail. This new class of lens antennas has a geometrical shape defined by the three-dimensional extension of Gielis' formula. The analytical description of the lens shape allows the development of a dedicated semianalytical hybrid modeling approach based on geometrical tube tracing and physical optic. In order to increase the accuracy of the model, the multiple reflections occurring within the lens are also taken into account.

\section{Introduction}

During the last years, lens antennas have attracted the attention of various researchers and companies because of their potential use in several application fields such as high frequency wireless communication systems $[1,2]$, millimeter wave imaging [3], space [4], smart antennas [5], radioastronomy [6], and radar systems [1]. In particular, in [1] a flat-top radiation pattern at $60 \mathrm{GHz}$ is achieved by considering a $2 \mathrm{D}$ arbitrary shape lens antenna close in two metal half disks useful for high speed Wi-Fi 802.1lad devices. Another application of such class of antennas is illustrated in [3] where the authors presented a lens-coupled patch antenna array for imaging applications. This architecture consists of a two-dimensional array featuring a monolithic microwave integrated circuit (MMIC) front-end. In [1] the authors presented a fully polarimetric integrated monopulse receiver working in $\mathrm{W}$-band frequency range. The proposed structure is composed by $2 \times 2$ array of slot-ring antennas covered by a hemispherical dielectric lens.

Lens antennas can be easily integrated in electronic circuits thanks to the relevant mechanical and thermal stability combined with the possibility of shaping the radiated beam by changing the lens geometry. During the last years, many research activities have been devoted to the development of 3D dielectric lenses characterized by canonical or axialsymmetrical geometry $[7,8]$ and only a few scientific studies have been dedicated to the analysis of more complex geometries [9-11]. In particular, in [10] the authors present a compact lens antenna with arbitrary shape working in millimeter wave frequency band, the design being carried out while neglecting the internal reflections occurring within the lens. However, in order to enhance the accuracy of the theoretical model based on geometrical optic (GO), it is essential to consider the effects of the internal reflections especially when high values of electric permittivity have to be used. To this end, in $[12,13]$ an accurate mathematical model is illustrated implementing the effects of the secondorder internal reflections for canonical lens geometry.

The aim of this work is to present, in detail, a semianalytical model to design a new class of shaped dielectric lens antennas whose geometry is described by Gielis' superformula $[14,15]$. The developed mathematical model is based on the tube tracing approximation [16]. The electromagnetic field inside the lens is evaluated by using the GO approximation and considering the effect of the internal reflections. 


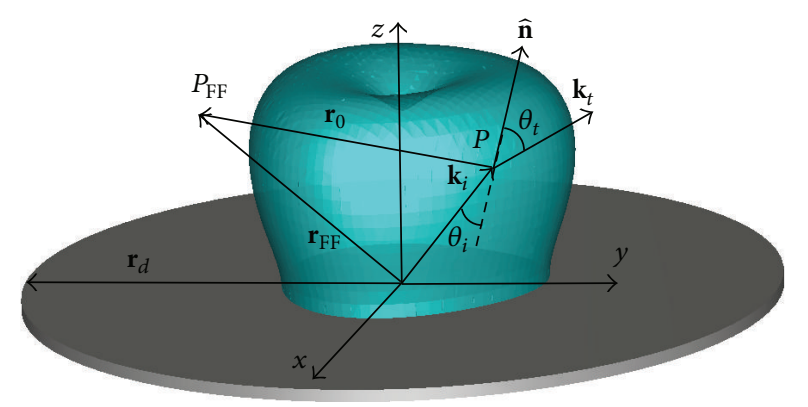

FIgURE 1: Geometrical structure of the electromagnetic system based on dielectric lens antenna.

In this way, an improvement of the numerical accuracy can be obtained. Moreover, the evaluation of the electromagnetic field radiated from the lens has been carried out applying the physical optics (PO) approximation. In particular, according to the equivalence principle, the radiation outgoing the lens is evaluated by the radiation of the equivalent electric and magnetic currents on the lens surface. The current distribution is calculated by making use of the Fresnel transmission coefficient on the lens surface in accordance with the GO procedure.

Thanks to Gielis' formulation it is possible to generate a wide range of $3 \mathrm{D}$ shapes in a simple and analytical way by changing a reduced number of parameters. The use of this formulation allows, also, an easy description of the geometrical characteristic of the antenna in terms of lens volume, area, curvature radius, and aspect ratio. Moreover, the analytical representation of the lens shape provides a number of benefits pertaining to the evaluation of the main physical quantities involved in the electromagnetic propagation equations. In this way, a reduced computation effort is required and more accurate results can be obtained.

\section{Mathematical Model}

The geometrical structure considered in the proposed electromagnetic model consists of a dielectric lens antenna placed on a perfectly electric conductor (PEC) circular plate having radius $r_{d}$ (see Figure 1 ).

The metal plate, acts as a ground plane and mechanical support of the radiating structure while reducing the backscattered radiation level. The lens is illuminated by the electromagnetic field emitted by a primary radio source such as open ended waveguide, patch antenna, horn antenna, or a coaxial probe integrated in the metal plate. The tube tracing approach based on the GO approximation is used to model the electromagnetic propagation inside the homogeneous dielectric lens. This approximation, allows a significant simplification of the mathematical model since it makes it possible to reduce the solution of Maxwell's equations to a geometrical problem. As a result, the adoption of such method allows the simulation of electrically large structures with a lower computational effort in comparison to full-wave numerical methods such as Finite-Difference-Time-Domain (FDTD) and Finite-Element-Method (FEM).

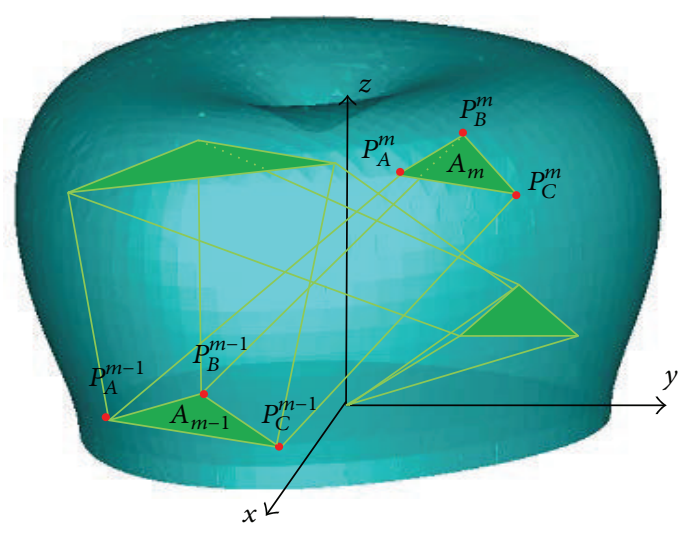

Figure 2: Area changes, from $A_{m-1}$ to $A_{m}$, of the tube surface during the propagation inside the lens.

The applicability condition of such method requires that the lens surface is placed within the far field zone of the feeding source and that the geometrical shape of the lens has a curvature radius larger than the operating wavelength. In this way, the travelling electromagnetic wave can be approximated by a set of tubes propagating over a rectilinear path inside the lens. Each tube has a triangular cross section with given surface area and is defined by three rays departing from each of the triangle vertices. The reflected and transmitted ray tubes are determined by using GO principles. As a result, the propagation path determines a change of the triangle area that leads a variation of the electromagnetic field amplitude in accordance with the power conservation law. In Figure 2, the geometrical change of the tube during the propagation inside the lens is illustrated. In particular, the surface area variation from $A_{m-1}$ to $A_{m}$ is given by triangle vertices intersection with the lens surface in points $P_{A}, P_{B}$, and $P_{C}$.

The surface area of each tube is evaluated by considering the overlap between the triangular surface, with vertices $P_{A}^{m}$, $P_{B}^{m}$, and $P_{C}^{m}$, and the elements of the tessellation of the lens surface. The amplitude of the electromagnetic field inside the tube surface is evaluated by using the Inverse Distance Weighting (IDW) algorithm. In particular, considering point $P$ inside of the triangle on the lens surface, the electric field amplitude is given by

$$
\mathbf{E}_{i}^{m}(P)=\frac{\mathbf{E}_{i}^{m}\left(P_{A}^{m}\right) u_{A}+\mathbf{E}_{i}^{m}\left(P_{B}^{m}\right) u_{B}+\mathbf{E}_{i}^{m}\left(P_{C}^{m}\right) u_{C}}{u_{A}+u_{B}+u_{C}},
$$

where

$$
\mathbf{E}_{i}^{m}\left(P_{k}^{m}\right)=\sqrt{\frac{A_{m}}{A_{m-1}}} \mathbf{E}_{i}^{m-1}\left(P_{k}^{m-1}\right) \quad k=A, B, C .
$$

$A_{m-1}$ and $A_{m}$ denote the area of the triangular section of the tube on the lens surface referring to $(m-1)$ th and $m$ th reflection order, respectively. However, in order to account for the changes of the tube cross section surface and to enforce the energy conservation law, the correction coefficient $\sqrt{A_{m} / A_{m-1}}$ is introduced. In (1), $P_{A}^{m}, P_{B}^{m}$, and $P_{C}^{m}$ denote the vertices of the surface intersected by the 
tube on the lens surface corresponding to $m$ th reflection order. The coefficients $u_{A}, u_{B}$, and $u_{C}$ are the inverse of the Euclidean distance between point $P$ and points $P_{A}^{m}, P_{B}^{m}$, and $P_{C}^{m}$, respectively.

According to the GO approach, the electric field transmitted outside the lens due to the direct ray and the internal reflections occurring inside the lens is given by the following equation:

$$
\mathbf{E}_{t}=\sum_{m} \mathbf{E}_{t}^{m},
$$

where the transmitted field outside the lens due to the $m$ th order internal reflection is

$$
\mathbf{E}_{t}^{m}=E_{t \|}^{m} \frac{\left(\widehat{\mathbf{n}} \times \widehat{\mathbf{k}}_{t}^{m}\right) \times \widehat{\mathbf{k}}_{t}^{m}}{\left\|\left(\widehat{\mathbf{n}} \times \widehat{\mathbf{k}}_{t}^{m}\right) \times \widehat{\mathbf{k}}_{t}^{m}\right\|}+E_{t \perp}^{m} \frac{\widehat{\mathbf{n}} \times \widehat{\mathbf{k}}_{t}^{m}}{\left\|\widehat{\mathbf{n}} \times \widehat{\mathbf{k}}_{t}^{m}\right\|} .
$$

In (4) $\widehat{\mathbf{n}}$ is the unit vector normal to the lens surface, $\widehat{\mathbf{k}}_{t}^{m}=\mathbf{k}_{t}^{m} /\left\|\mathbf{k}_{t}^{m}\right\|$ is the transmitted unit wave vector due to the $m$ th internal reflection with $\left\|\mathbf{k}_{t}^{m}\right\|=2 \pi n_{0} / \lambda_{0}$, where $n_{0}$ is the refractive index of the external medium, and $\lambda_{0}$ is the free space wavelength. The orthogonal $E_{t \perp}^{m}$ and parallel components $E_{t \|}^{m}$ of the transmitted electric field are determined by multiplying the orthogonal $E_{i \perp}^{m}$ and parallel $E_{i \|}^{m}$ components of the incident electric field $\mathbf{E}_{i}^{m}$ in such point by the proper Fresnel transmission coefficient. The evaluation of the incident components of the electric field is carried out by means of the following equations:

$$
\begin{aligned}
E_{i \perp}^{m} & =\mathbf{E}_{i}^{m} \cdot \frac{\widehat{\mathbf{n}} \times \widehat{\mathbf{k}}_{i}^{m}}{\left\|\widehat{\mathbf{n}} \times \widehat{\mathbf{k}}_{i}^{m}\right\|}, \\
E_{i \|}^{m} & =\mathbf{E}_{i}^{m} \cdot \frac{\left(\widehat{\mathbf{n}} \times \widehat{\mathbf{k}}_{i}^{m}\right) \times \widehat{\mathbf{k}}_{i}^{m}}{\left\|\left(\widehat{\mathbf{n}} \times \widehat{\mathbf{k}}_{i}^{m}\right) \times \widehat{\mathbf{k}}_{i}^{m}\right\|},
\end{aligned}
$$

where $\widehat{\mathbf{k}}_{i}^{m}=\mathbf{k}_{i}^{m} /\left\|\mathbf{k}_{i}^{m}\right\|$ is the incident unit wave vector due to $m$ th internal reflection with $\left\|\mathbf{k}_{i}^{m}\right\|=2 \pi n_{d} / \lambda_{0}$, where $n_{d}$ is the refractive index of the homogeneous dielectric material forming the lens.

In case of direct tube, $m=1$, the incident field $\mathbf{E}_{i}^{m}\left(P_{m}\right)$ at point $P_{m}$ is calculated using the far field pattern of the source and instead, for $m>1, \mathbf{E}_{i}^{m}\left(P_{m}\right)$ is derived from $(m-1)$ th reflected wave contribution as

$$
\mathbf{E}_{i}^{m}\left(P_{m}\right)=\mathbf{E}_{r}^{m-1}\left(P_{m-1}\right) e^{j\left\|\mathbf{k}_{i}^{m}\right\| d_{m}},
$$

where $d_{m}$ is the path length between observation point $P_{m}$ and point $P_{m-1}$, where the reflection takes place. The reflected field $\mathbf{E}_{r}^{m-1}\left(P_{m-1}\right)$ appearing in (7) is given by the following equation

$$
\begin{aligned}
\mathbf{E}_{r}^{m-1}\left(P_{m-1}\right)= & E_{r \|}^{m-1} \frac{\left[\widehat{\mathbf{k}}_{r}^{m-1} \times(-\widehat{\mathbf{n}})\right] \times \widehat{\mathbf{k}}_{r}^{m-1}}{\left\|\left[\widehat{\mathbf{k}}_{r}^{m-1} \times(-\widehat{\mathbf{n}})\right] \times \widehat{\mathbf{k}}_{r}^{m-1}\right\|} \\
& +E_{r \perp}^{m-1} \frac{\widehat{\mathbf{k}}_{r}^{m-1} \times(-\widehat{\mathbf{n}})}{\left\|\widehat{\mathbf{k}}_{r}^{m-1} \times(-\widehat{\mathbf{n}})\right\|},
\end{aligned}
$$

where parallel $E_{r \|}^{m-1}$ and orthogonal $E_{r \perp}^{m-1}$ electric field components of $(m-1)$ th reflected wave are obtained by multiplying the corresponding components with the Fresnel reflection coefficient. $\widehat{\mathbf{k}}_{i}^{m}=\mathbf{k}_{i}^{m} /\left\|\mathbf{k}_{i}^{m}\right\|$ term is the reflected unit vector referring to $(m-1)$ th internal reflection at point $P_{m-1}$.

In order to evaluate the transmitted and reflected unit wave vector, Snell's law at the interface between the dielectric lens and the outside medium has to be considered. In particular, the transmission and reflection phenomena are modeled by the following relations:

$$
\begin{aligned}
n_{d} \widehat{\mathbf{k}}_{i} \times \widehat{\mathbf{n}} & =n_{0} \widehat{\mathbf{k}}_{t} \times \widehat{\mathbf{n}}, \\
\widehat{\mathbf{k}}_{i} \times \widehat{\mathbf{n}} & =\widehat{\mathbf{k}}_{r} \times(-\widehat{\mathbf{n}}),
\end{aligned}
$$

where the transmitted unit wave vector $\widehat{\mathbf{k}}_{t}$, the reflected unit wave vector $\widehat{\mathbf{k}}_{r}$, the tangential unit vector $\widehat{\mathbf{t}}_{\text {, and the normal }}$ unit vector $\widehat{\mathbf{n}}$ to the lens surface are given by

$$
\begin{aligned}
\widehat{\mathbf{k}}_{t}(\theta, \phi)= & \cos \left(\theta_{t}\right) \widehat{\mathbf{n}}+\sin \left(\theta_{t}\right) \widehat{\mathbf{t}} \\
= & {\left[\cos \left(\theta_{t}\right) n_{r}+\sin \left(\theta_{t}\right) t_{r}\right] \widehat{\mathbf{r}} } \\
& +\left[\cos \left(\theta_{t}\right) n_{\theta}+\sin \left(\theta_{t}\right) t_{\theta}\right] \widehat{\boldsymbol{\theta}} \\
& +\left[\cos \left(\theta_{t}\right) n_{\varphi}+\sin \left(\theta_{t}\right) t_{\varphi}\right] \widehat{\boldsymbol{\varphi}}, \\
\widehat{\mathbf{k}}_{r} \times \widehat{\mathbf{n}}= & \left(\sin \theta_{i}-\cos \theta_{i}\right) \widehat{\mathbf{n}}, \\
\widehat{\mathbf{t}}= & \frac{-\widehat{\mathbf{n}} \times\left(\widehat{\mathbf{n}} \times \widehat{\mathbf{k}}_{i}\right)}{\left\|\widehat{\mathbf{n}} \times\left(\widehat{\mathbf{n}} \times \widehat{\mathbf{k}}_{i}\right)\right\|}, \\
\widehat{\mathbf{n}}= & \frac{\partial \mathbf{r} / \partial \theta \times \partial \mathbf{r} / \partial \varphi}{\|\partial \mathbf{r} / \partial \theta \times \partial \mathbf{r} / \partial \varphi\|},
\end{aligned}
$$

where the normal unit vector $\widehat{\mathbf{n}}=n_{r} \widehat{\mathbf{r}}+n_{\theta} \widehat{\boldsymbol{\theta}}+n_{\varphi} \widehat{\boldsymbol{\varphi}}$ and tangential unit vector $\widehat{\mathbf{t}}=t_{r} \widehat{\mathbf{r}}+t_{\theta} \widehat{\boldsymbol{\theta}}+t_{\varphi} \widehat{\boldsymbol{\varphi}}$ to the lens surface in spherical coordinates are introduced. The terms $\theta_{t}$ and $\theta_{i}$ denote the transmitted and incident angle formed by the wave propagation vector and the normal unit vector to the surface in a given point, respectively:

$$
\begin{aligned}
& \theta_{i}=\tan ^{-1}\left(\frac{\widehat{\mathbf{k}}_{i} \cdot \widehat{\mathbf{t}}}{\widehat{\mathbf{k}}_{i} \cdot \widehat{\mathbf{n}}}\right), \\
& \theta_{t}=\sin ^{-1}\left(n_{d} \sin \theta_{i}\right) .
\end{aligned}
$$

Once the transmitted and incident angles are computed, the Fresnel coefficients for the transmitted wave component can be evaluated as

$$
\begin{aligned}
t_{\perp} & =\frac{2 n_{d} \cos \theta_{i}}{n_{d} \cos \theta_{i}+n_{0} \cos \theta_{t}}, \\
t_{\|} & =\frac{2 n_{d} \cos \theta_{i}}{n_{d} \cos \theta_{t}+n_{0} \cos \theta_{i}}
\end{aligned}
$$


and the reflected component of the electric field is

$$
\begin{aligned}
r_{\perp} & =\frac{n_{d} \cos \theta_{t}-n_{0} \cos \theta_{i}}{n_{d} \cos \theta_{t}+n_{0} \cos \theta_{t}}, \\
r_{\|} & =\frac{n_{d} \cos \theta_{i}-n_{0} \cos \theta_{t}}{n_{d} \cos \theta_{i}+n_{0} \cos \theta_{t}} .
\end{aligned}
$$

Vector $\mathbf{r}=r \widehat{\mathbf{r}}$ appearing in (13) is the radius vector describing the lens surface defined by Gielis' superformula as follows [17, 18]:

$$
\mathbf{r}=\widehat{\mathbf{r}} \sqrt{z^{2}+y^{2}+x^{2}}
$$

with

$$
\begin{aligned}
x(\psi, \zeta) & =R_{\psi} \cos (\psi) R_{\zeta} \cos (\zeta), \\
y(\psi, \zeta) & =R_{\psi} \sin (\psi) R_{\zeta} \cos (\zeta), \\
z(\psi, \zeta) & =R_{\zeta} \sin (\zeta), \\
R_{\psi} & =\left[\left|\frac{\cos \left(m_{1} \psi / 4\right)}{a_{1}}\right|^{n_{1}}+\left|\frac{\sin \left(m_{2} \psi / 4\right)}{a_{2}}\right|^{n_{2}}\right]^{-1 / b_{1}}, \\
R_{\zeta} & =\left[\left|\frac{\cos \left(m_{3} \zeta / 4\right)}{a_{3}}\right|^{n_{3}}+\left|\frac{\sin \left(m_{4} \zeta / 4\right)}{a_{4}}\right|^{n_{4}}\right]^{-1 / b_{2}},
\end{aligned}
$$

where $n_{p}, a_{p}, m_{p}, p=1 \cdots 4$, and $b_{q}, q=1,2$, are real-valued parameters selected in such a way that the surface of the lens is actually closed and characterized, at any point, by curvature radius larger than the working wavelength in accordance with the GO approximation. The parameters $\zeta \in[0, \pi / 2]$ and $\psi \epsilon$ $[-\pi, \pi]$ denote real-valued parameters appearing in Gielis' formula, whereas the spherical angles $\theta$ and $\varphi$ are obtained by means of the following relations:

$$
\begin{aligned}
& \theta=\cos ^{-1}\left(\frac{z}{r}\right), \\
& \varphi=\tan ^{-1}\left(\frac{y}{x}\right) .
\end{aligned}
$$

The derivatives of the radius vector $\mathbf{r}$ appearing in (13) are given by

$$
\begin{aligned}
& \frac{\partial \mathbf{r}}{\partial \theta}=\frac{\partial r}{\partial \theta} \widehat{\mathbf{r}}+r \widehat{\boldsymbol{\theta}}, \\
& \frac{\partial \mathbf{r}}{\partial \varphi}=\frac{\partial r}{\partial \varphi} \widehat{\mathbf{r}}+r \sin (\theta) \widehat{\boldsymbol{\varphi}},
\end{aligned}
$$

where

$$
\begin{aligned}
\frac{\partial r}{\partial \theta} & =\frac{\partial r}{\partial \zeta} \frac{\partial \zeta}{\partial \theta}, \\
\frac{\partial r}{\partial \varphi} & =\frac{\partial r}{\partial \psi}-\tan \gamma \frac{\partial r}{\partial \theta}, \\
\gamma & =\tan ^{-1}\left(\frac{\partial \theta}{\partial \psi}\right) .
\end{aligned}
$$

According to Gielis' description of the lens surface, the derivatives of $\mathbf{r}$ with respect to $\zeta$ appearing in (21) can be evaluated as follows:

$$
\begin{aligned}
& \frac{\partial r}{\partial \zeta}=\frac{1}{r}\left(x \frac{\partial x}{\partial \zeta}+y \frac{\partial y}{\partial \zeta}+z \frac{\partial z}{\partial \zeta}\right) \\
& \frac{\partial \zeta}{\partial \theta}=\left[\frac{r(d z / d \zeta)-z(d r / d \zeta)}{r^{2} \sqrt{1-(z / r)^{2}}}\right]^{-1}
\end{aligned}
$$

with

$$
\begin{aligned}
& \frac{d z}{d \zeta}=\frac{d R_{\zeta}}{d \zeta} \sin \zeta+R_{\zeta} \cos \zeta, \\
& \frac{d y}{d \zeta}=R_{\psi} \frac{d R_{\zeta}}{d \zeta} \cos \zeta \sin \psi-R_{\psi} R_{\zeta} \sin \zeta \sin \psi, \\
& \frac{d x}{d \zeta}=R_{\psi} \frac{d R_{\zeta}}{d \zeta} \cos \zeta \cos \psi-R_{\psi} R_{\zeta} \sin \zeta \cos \psi, \\
& \frac{\partial R_{\zeta}}{\partial \zeta}=-\frac{1}{b_{2}}\left[\left|\frac{\cos \left(m_{3} \zeta / 4\right)}{a_{3}}\right|^{n_{3}}\right. \\
& \left.+\left|\frac{\sin \left(m_{4} \zeta / 4\right)}{a_{4}}\right|^{n_{4}}\right]^{-\left(1+b_{2}\right) / b_{2}} \\
& \left.\quad \cdot\left|n_{3}\right| \frac{\cos \left(m_{3} \zeta / 4\right)}{a_{3}}\right|^{n_{3}-1} \frac{\partial}{\partial \zeta}\left|\frac{\cos \left(m_{3} \zeta / 4\right)}{a_{3}}\right| \\
& \left.\quad+n_{4}\left|\frac{\sin \left(m_{4} \zeta / 4\right)}{a_{4}}\right|^{n_{4}-1} \frac{\partial}{\partial \zeta}\left|\frac{\sin \left(m_{4} \zeta / 4\right)}{a_{4}}\right|\right],
\end{aligned}
$$

$$
\begin{aligned}
& \frac{\partial}{\partial \zeta}\left|\frac{\cos \left(m_{3} \zeta / 4\right)}{a_{3}}\right|=-\frac{m_{3}}{4 a_{3}} \sin \left(\frac{m_{3} \zeta}{4}\right)\left[2 H\left(a_{3}\right)-1\right] \\
& \cdot\left[2 H\left(\left|\frac{m_{3} \zeta}{4}\right|_{2 \pi}-\frac{3}{4} \pi\right)-2 H\left(\left|\frac{m_{3} \zeta}{4}\right|_{2 \pi}-\frac{\pi}{2}\right)\right. \\
& \quad+1], \\
& \frac{\partial}{\partial \zeta}\left|\frac{\sin \left(m_{4} \zeta / 4\right)}{a_{4}}\right|=\frac{m_{4}}{4 a_{4}} \cos \left(\frac{m_{4} \zeta}{4}\right)\left[2 H\left(a_{4}\right)-1\right][1 \\
& \left.\quad-2 H\left(\left|\frac{m_{4} \zeta}{4}\right|_{2 \pi}-\pi\right)\right],
\end{aligned}
$$

where $H(\cdot)$ denotes the Heaviside function.

The derivative of $\mathbf{r}$ with respect to $\psi$ appearing in (22) can be evaluated as follows:

$$
\frac{\partial r}{\partial \psi}=\frac{1}{r}\left(x \frac{\partial x}{\partial \psi}+y \frac{\partial y}{\partial \psi}\right)
$$




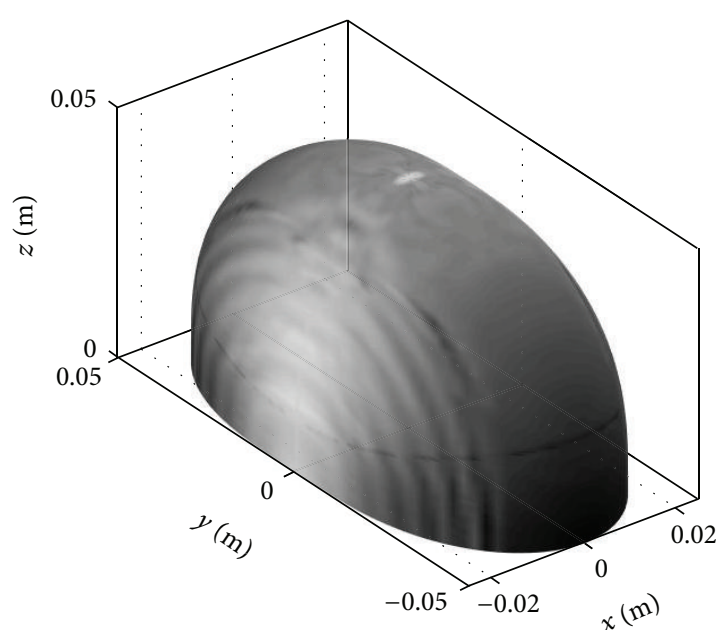

(a)

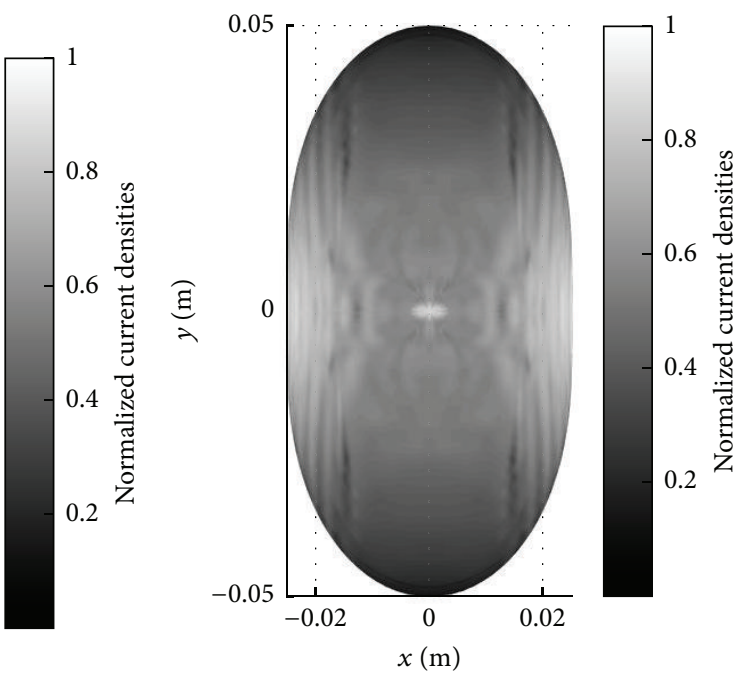

(b)

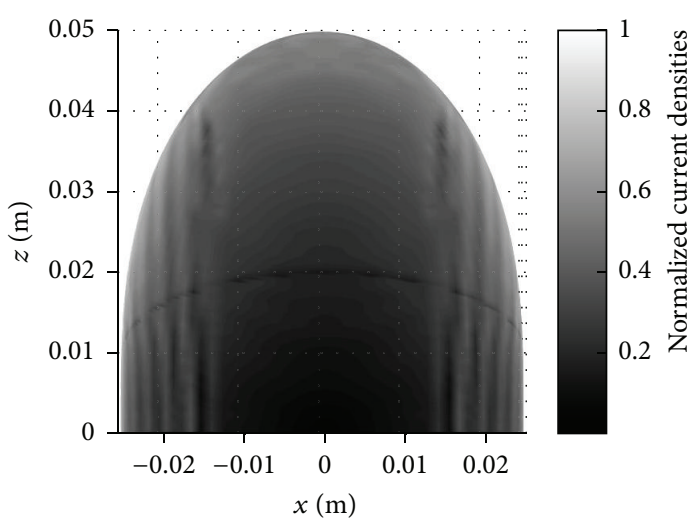

(d)

FIGURE 3: Densities current distribution on the lens surface considering $m=2$ internal reflections: (a) prospective view, (b) top view, and (c, d) lateral views.

where

$$
\begin{aligned}
\frac{d y}{d \psi} & =R_{\zeta} \frac{d R_{\psi}}{d \psi} \cos \zeta \sin \psi+R_{\psi} R_{\zeta} \cos \zeta \cos \psi, \\
\frac{d x}{d \psi} & =R_{\zeta} \frac{d R_{\psi}}{d \psi} \cos \zeta \cos \psi-R_{\psi} R_{\zeta} \cos \zeta \sin \psi, \\
\frac{\partial R_{\psi}}{\partial \psi} & =-\frac{1}{b_{1}}\left[\left|\frac{\cos \left(m_{1} \psi / 4\right)}{a_{1}}\right|^{n_{1}}\right. \\
+ & \left.\left|\frac{\sin \left(m_{2} \psi / 4\right)}{a_{2}}\right|^{n_{2}}\right]^{-\left(1+b_{1}\right) / b_{1}} \\
& \cdot\left[n_{1}\left|\frac{\cos \left(m_{1} \psi / 4\right)}{a_{1}}\right|^{n_{1}-1} \frac{\partial}{\partial \zeta}\left|\frac{\cos \left(m_{1} \psi / 4\right)}{a_{1}}\right|\right. \\
+ & \left.n_{2}\left|\frac{\sin \left(m_{2} \psi / 4\right)}{a_{2}}\right|^{n_{2}-1} \frac{\partial}{\partial \zeta}\left|\frac{\sin \left(m_{2} \psi / 4\right)}{a_{2}}\right|\right],
\end{aligned}
$$

$$
\begin{aligned}
& \frac{\partial}{\partial \psi}\left|\frac{\cos \left(m_{1} \psi / 4\right)}{a_{1}}\right|=-\frac{m_{1}}{4 a_{1}} \sin \left(\frac{m_{1} \psi}{4}\right)\left[2 H\left(a_{1}\right)-1\right] \\
& \cdot\left[2 H\left(\left|\frac{m_{1} \psi}{4}\right|_{2 \pi}-\frac{3}{4} \pi\right)-2 H\left(\left|\frac{m_{1} \psi}{4}\right|_{2 \pi}-\frac{\pi}{2}\right)\right. \\
& +1], \\
& \frac{\partial}{\partial \psi}\left|\frac{\sin \left(m_{2} \psi / 4\right)}{a_{2}}\right|=\frac{m_{2}}{4 a_{2}} \cos \left(\frac{m_{2} \psi}{4}\right)\left[2 H\left(a_{2}\right)-1\right][1 \\
& \left.-2 H\left(\left|\frac{m_{2} \psi}{4}\right|_{2 \pi}-\pi\right)\right], \\
& \frac{\partial \theta}{\partial \psi}=\frac{z}{r^{2}}\left[\sqrt{1-\left(\frac{z}{r}\right)}\right]^{-1 / 2} \frac{\partial r}{\partial \psi} .
\end{aligned}
$$

Once the contribution of all internal reflections has been evaluated by the GO method, the equivalent electric $\mathbf{J}_{s}$ and magnetic $\mathbf{M}_{s}$ current densities excited along the surface of the lens can be easily determined. According to the PO 


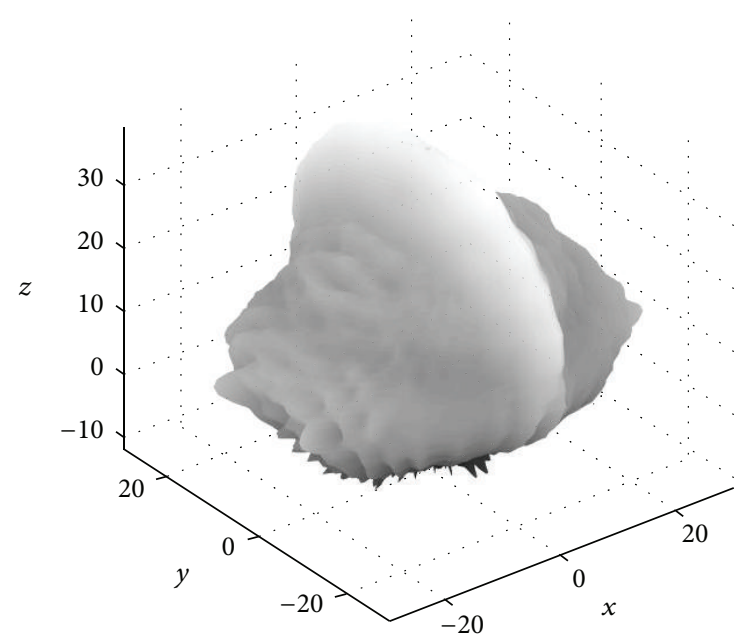

(a)

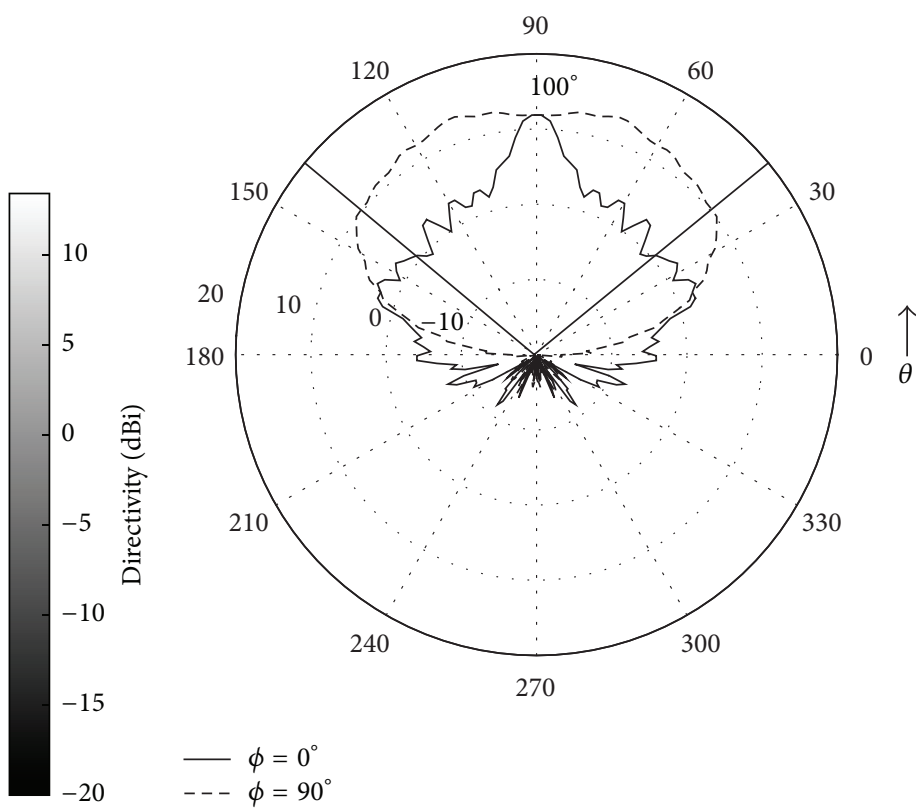

(b)

Figure 4: Radiation solid (a) and polar sections (b) with $\phi=0^{\circ}$ solid line and $\phi=90^{\circ}$ dashed line of the considered lens antenna.

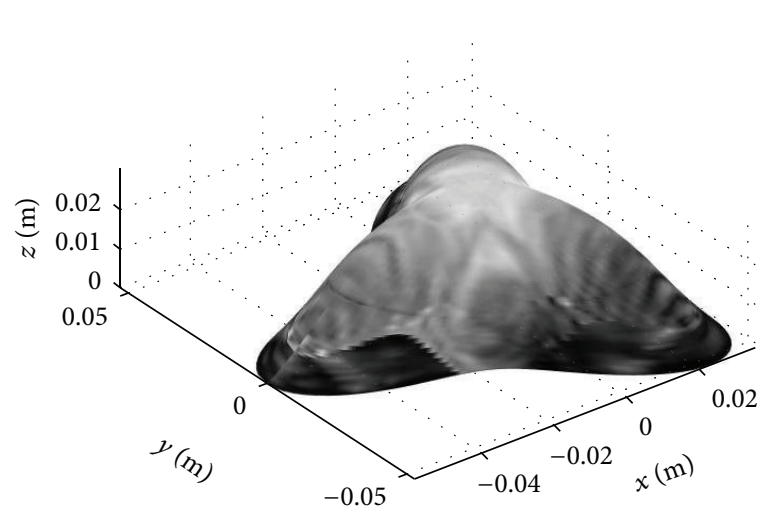

(a)

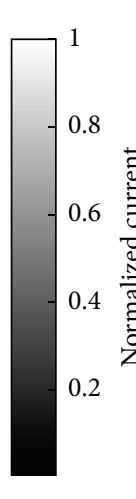

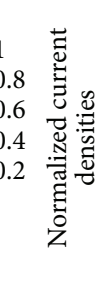

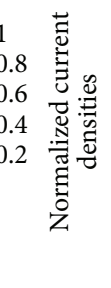

(c)

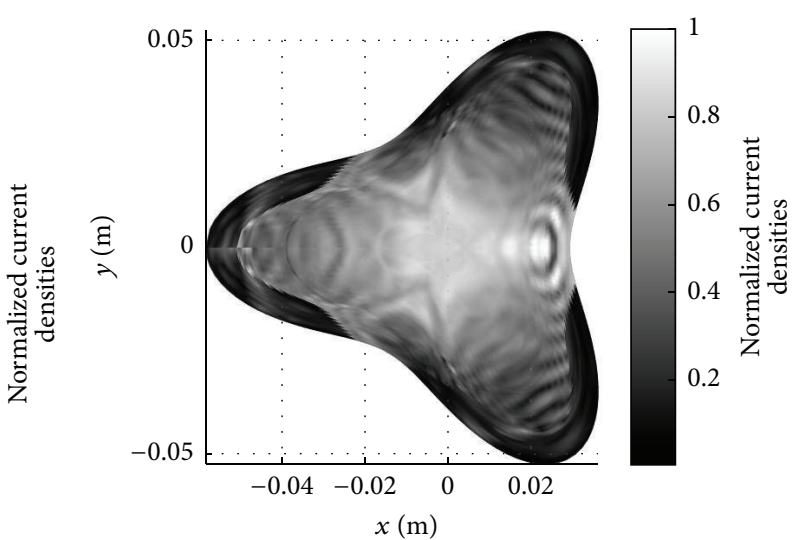

(b)

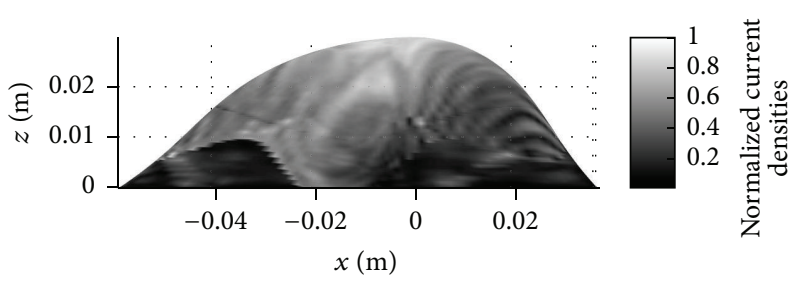

(d)

Figure 5: Densities current distribution on the lens surface considering $m=3$ internal reflection: (a) prospective view, (b) top view, and (c, d) lateral views.

approximation, the electromagnetic field radiated in the spatial domain outside the lens at the general observation point $P_{\mathrm{FF}}=\left(\mathbf{r}_{\mathrm{FF}}, \theta_{\mathrm{FF}}, \varphi_{\mathrm{FF}}\right)$ can be computed by the following integral expression:

$$
\begin{aligned}
& \mathbf{E}_{\mathrm{FF}}\left(P_{\mathrm{FF}}\right) \\
& \quad=\mathbf{j} \frac{e^{-\mathbf{j} k_{0} \mathbf{r}_{\mathrm{FF}}}}{2 \lambda_{0} \mathbf{r}_{\mathrm{FF}}} \int_{S}\left[\eta_{0} \mathbf{J}_{S} \times \widehat{\mathbf{u}}_{0}-\mathbf{M}_{S}\right] \times \widehat{\mathbf{u}}_{0} \mathrm{e}^{\mathrm{j} k_{0} \mathbf{r}_{0} \cdot \widehat{\mathbf{u}}_{0}} d S,
\end{aligned}
$$




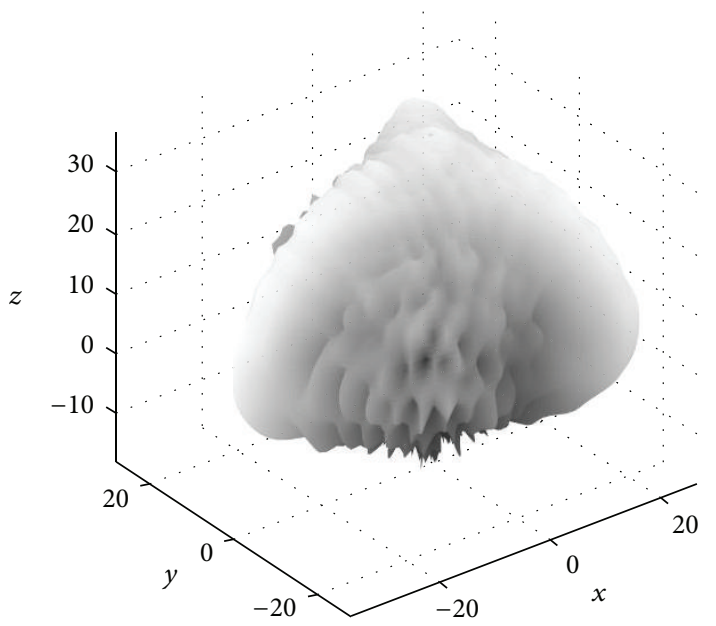

(a)

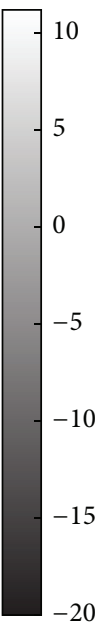

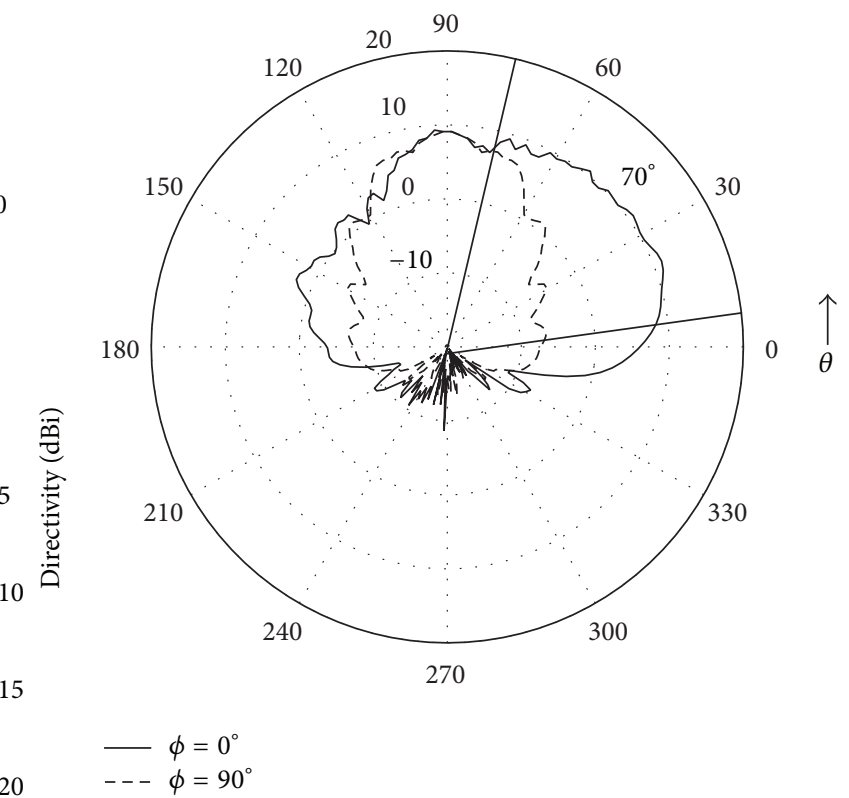

(b)

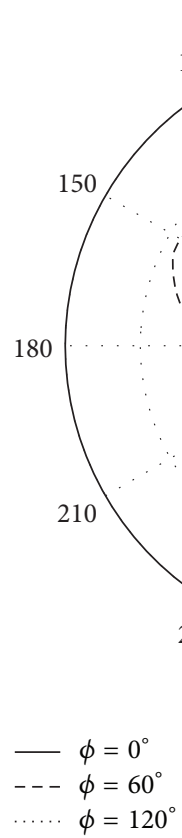

90

20

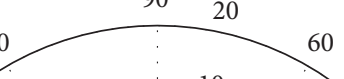

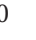




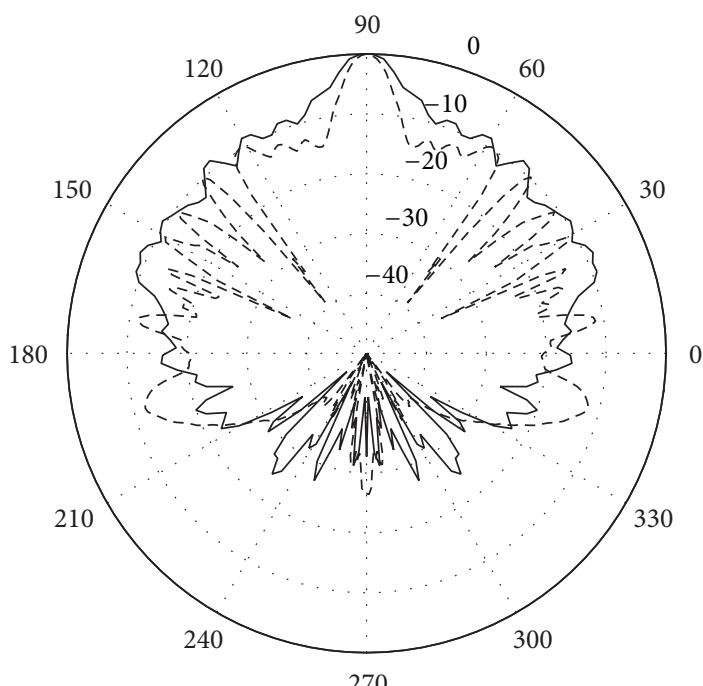

$\begin{array}{ll}--- & \text { Full wave } \\ & \text { GO/PO approach }\end{array}$

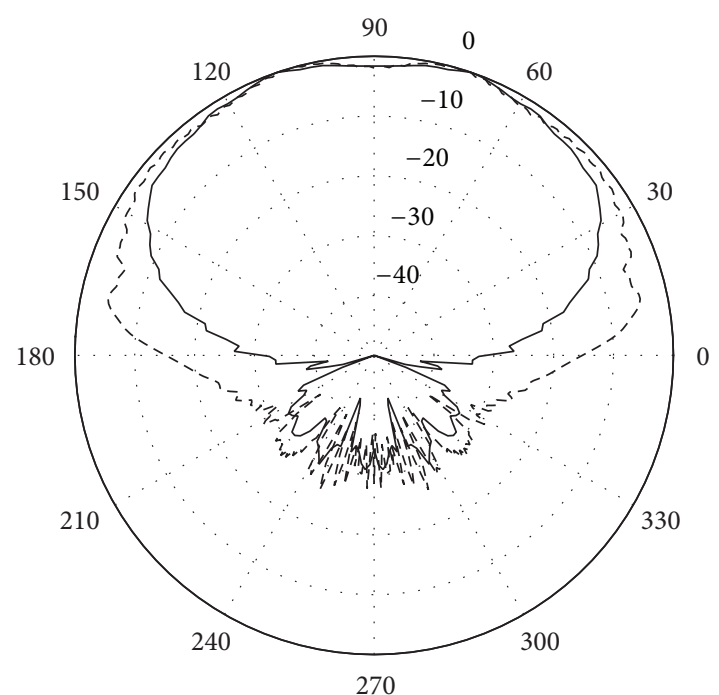

- - - Full wave

$\mathrm{GO} / \mathrm{PO}$ approach

(a)

(b)

FIgURE 7: Comparison between the normalized directivity of the \#LA1 lens antenna: polar sections with (a) $\phi=0^{\circ}$ and (b) $\phi=90^{\circ}$.

Furthermore, the electromagnetic radiation properties can be tuned by changing the lens parameters in order to synthesize a desired radiation pattern. Moreover, Gielis' superformula is useful for defining analytically all the physical quantities involved in the electromagnetic problem. This in turn is beneficial for enhancing the accuracy of the results and, at the same time, reducing the computational cost of the analysis in comparison to a brute-force numerical approach.

The developed modeling technique is adopted to design a particular lens antenna showing a flat-top radiating pattern at frequency $f=60 \mathrm{GHz}$. This type of antenna could be integrated in communication systems covering a wide area. The proposed lens (\#LA1) is made out of a dielectric material with refractive index equal to $n_{d}=1.42$, having a minimum radius of $r_{\min }=25 \mathrm{~mm}$ and described by the following parameters of Gielis' superformula: $n_{i}=4$, $a_{i}=1, m_{i}=2, i=1 \cdots 4$, and $b_{1}=b_{2}=1$. The selected Gielis' parameters ensure a curvature radius of the lens larger than the operating wavelength in all the points along the lens surface, in accordance with the applicability restrictions of the GO approximation. The lens is placed on a metal disk plate with radius $r_{d}=100 \mathrm{~mm}$ and fed by an open ended circular waveguide with diameter $a_{g}=2.3 \mathrm{~mm}$, filled up by the same dielectric material forming the lens and positioned at center of such metallic plate. The numerical simulation has been performed considering a lens surface tessellation composed by $160 \times 160$ elements and $m=2$ internal reflections. Figure 3 shows the equivalent electric current densities distribution on the lens surface considering the internal reflection contribution.

Figure 4 illustrates (a) the radiation solids (far field pattern) and (b) the polar sections generated by the densities current pattern illustrated in Figure 3. It is worth noting that the antenna main lobe is characterized by an angular aperture at $-3 \mathrm{~dB}$ of about 100 degrees with a peak directivity value of about $13 \mathrm{dBi}$.

Another numerical example relevant to the design of a lens antenna characterized by a triple radiation beam pattern at frequency $f=60 \mathrm{GHz}$ is here reported. This type of antenna could be adopted in communication systems implementing a spatial-division multiplexing useful for increasing the channel capacity where the position of the receiver is known. In this case, the proposed lens (\#LA2) is made out of a dielectric material with refractive index equal to $n_{d}=1.42$, having a minimum radius of $r_{\min }=25 \mathrm{~mm}$ and described by the following Gielis' parameters: $n_{1}=n_{2}=4, n_{3}=3, n_{4}=1$, $a_{i}=1$, and $m_{i}=3(i=1 \cdots 4)$. Also in this case, the selected Gielis' parameters ensure a minimum curvature radius of the lens surface that satisfies the applicability conditions of the GO approximation. A metal disk plate with radius $r_{d}=$ $100 \mathrm{~mm}$ is also considered and the feeding source consists of an open ended circular waveguide having diameter $a_{g}=$ $2.7 \mathrm{~mm}$ and filled up by the same dielectric material forming the lens. Also, in this case, the lens surface tessellation is composed by $160 \times 160$ elements and $m=3$ internal reflections have been taken into account. In Figure 5 the distribution of the equivalent electric current densities on the lens surface is shown. The internal reflections are properly taken into account.

Figure 6 shows (a) the radiation solids and ((b), (c)) polar sections, generated by the current density distribution illustrated in Figure 5. It is possible to observe three main lobes having an angular aperture at $-3 \mathrm{~dB}$ of about 70 degrees pointing in three different directions $\phi=0^{\circ}, \phi=120^{\circ}$, and $\phi=240^{\circ}$ with a directivity of about $11 \mathrm{dBi}$ and an elevation angle of $\theta=45^{\circ}$.

Both the illustrated lens antenna structures feature specific properties in terms of radiation pattern that can be 


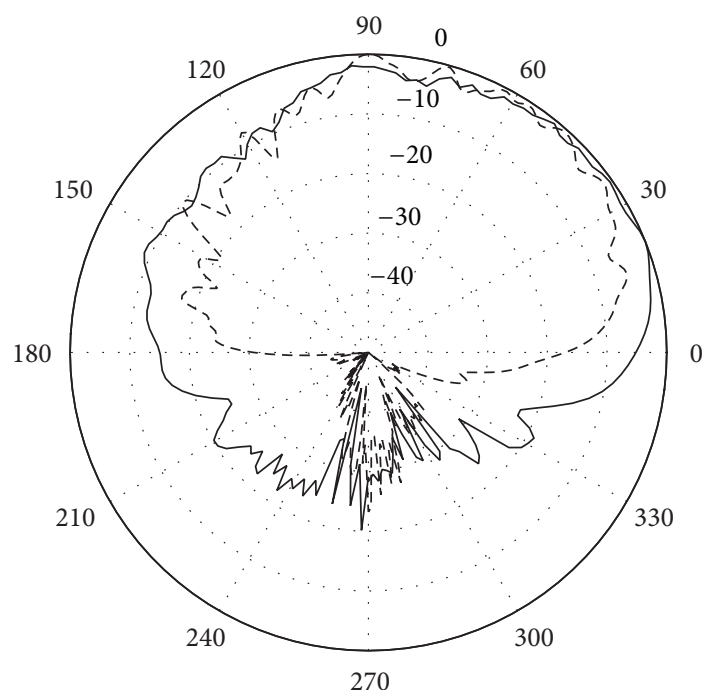

- - - Full wave

— GO/PO approach

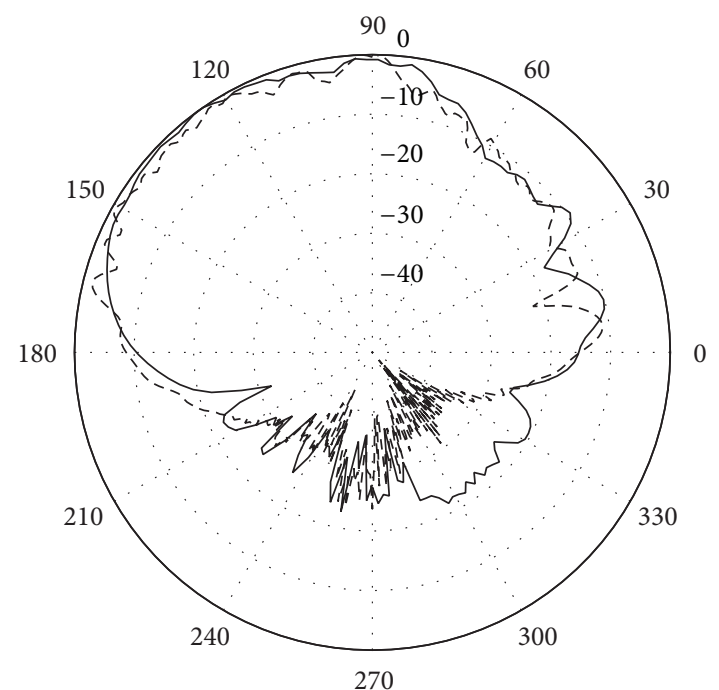

- - Full wave

— GO/PO approach

(a)

(b)

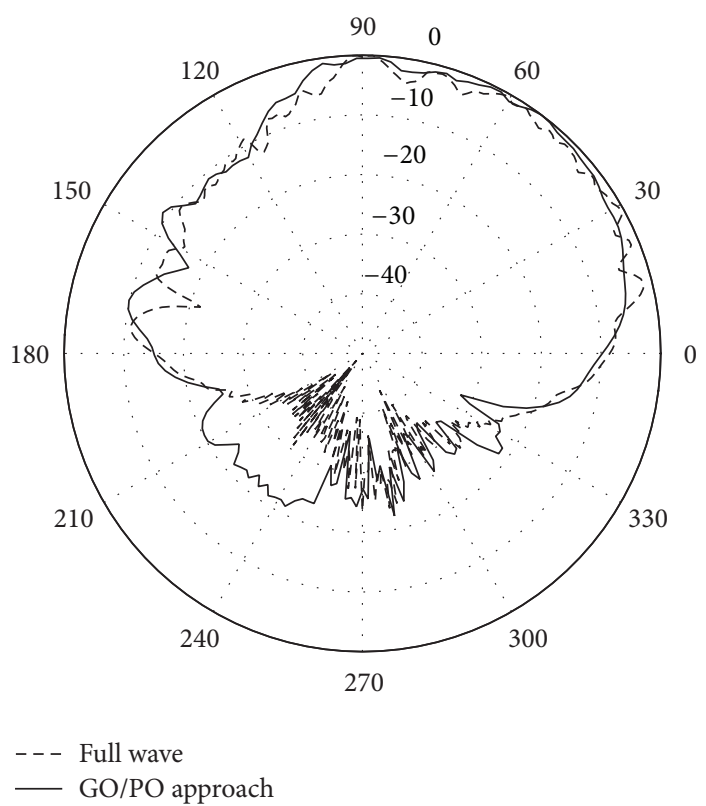

(c)

FIGURE 8: Comparison between the normalized directivity of the \#LA2 lens antenna: polar sections with (a) $\phi=0^{\circ},(\mathrm{b}) \phi=60^{\circ}$, and (c) $\phi=120$.

beneficial for the newly introduced Wi-Fi 802.11ad communication protocol working at frequency $f=60 \mathrm{GHz}$. Moreover, the obtained numerical results confirm the feasibility of the proposed mathematical model to study the special class of lens antennas defined by Gielis' superformula.

\section{Model Validation}

In order to validate the developed modeling approach, a comparison with a commercially available full-wave electromagnetic solver CST Microwave Studio has been carried out.
Figures 7 and 8 show the normalized directivity of the lens antennas \#LA1 and \#LA2, respectively, as computed by the developed GO/PO asymptotic approach and the commercial software. It is worthwhile to note that, within the angular width of the main lobe (antenna beam width or half-power beam width, more important for antenna design), a closer agreement between the full-wave and GO-PO numerical results has been obtained. Outside such angular region (less important for antenna design), an accuracy decrease of the GO-PO results can be observed. This occurrence, can be explained taking into account that the source field used in 
GO-PO approach is slightly different from that resulting from the full-wave method. In fact, in GO-PO approach the incident electromagnetic field on the lens surface corresponds to the far field pattern from a circular aperture on an infinite metallic plate. Instead, in the full-wave method the calculation of the incident electromagnetic field takes into account the finite size of the metallic plate. However, the full-wave analysis requires more computational resources in terms of memory occupation and simulation time. As a matter of fact, using a workstation with dual Intel Xeon E5645 processor, frequency of $2.4 \mathrm{GHz}$, the computational time and memory allocation required by the full-wave solver are about $15 \mathrm{hr}$ and 22 GBytes, respectively, for lens \#LA1 and about $10 \mathrm{hr}$ and 15 GBytes, respectively, for lens \#LA2. On the other hand, the developed GO-PO procedure is characterized by a computational time and memory allocation of about $3 \mathrm{hr}$ and 2.5 GBytes, on the same workstation.

\section{Conclusion}

A novel mathematical procedure based on GO/PO tube tracing approach for modeling the electromagnetic propagation inside the lens antenna has been illustrated. The proposed model is applied to a special class of lens antennas having the three-dimensional shape defined by Gielis' superformula. The analytical expression of the lens surface reduces the drawbacks due to various numerical approximations while ensuring more accurate results. The applicability of the proposed model could be extended to design complex shaped lens antennas for a wide range of applications covering both the microwave and optical frequency band. A proper selection of Gielis' shape allows the performance improvement of the antenna in terms of directivity, multibeam radiating shape, beam-steering angle, beam stability, wide beam angle, and radiating efficiency.

\section{Conflict of Interests}

The authors declare that there is no conflict of interests regarding the publication of this paper.

\section{References}

[1] S. Raman, N. S. Barker, and G. M. Rebeiz, "A W-band dielectriclens-based integrated monopulse radar receiver," IEEE Transactions on Microwave Theory and Techniques, vol. 46, no. 12, pp. 2308-2316, 1998.

[2] A. Bisognin, D. Titz, F. Ferrero et al., “3D printed plastic $60 \mathrm{GHz}$ lens: enabling innovative millimeter wave antenna solution and system," in Proceedings of the IEEE MTT-S International Microwave Symposium (IMS '14), pp. 1-4, Tampa, Fla, USA, June 2014.

[3] K. Uehara, K. Miyashita, K.-I. Natsume, K. Hatakeyama, and K. Mizuno, "Lens-coupled imaging arrays for the millimeter- and submillimeter-wave regions," IEEE Transactions on Microwave Theory and Techniques, vol. 40, no. 5, pp. 806-811, 1992.

[4] O. Yurduseven, N. Llombart, A. Neto, and J. Baselmans, "A dual polarized antenna for $\mathrm{THz}$ space applications: antenna design and lens optimization," in Proceedings of the IEEE Antennas and
Propagation Society International Symposium (APSURSI'14), pp. 191-192, IEEE, Memphis, Tenn, USA, July 2014.

[5] S. Ravishankar and B. Dharshak, "A rapid direction of arrival estimation procedure for adaptive array antennas covered by a shaped dielectric lens," in Proceedings of the IEEE Radio and Wireless Symposium (RWS '14), pp. 124-126, Newport Beach, Calif, USA, January 2014.

[6] T. H. Buttgenbach, "An improved solution for integrated array optics in quasi-optical $\mathrm{mm}$ and submm receivers: the hybrid antenna," IEEE Transactions on Microwave Theory and Techniques, vol. 41, no. 10, pp. 1750-1761, 1993.

[7] D. F. Filipovic and G. M. Rebeiz, "Double-slot antennas on extended hemispherical and elliptical quartz dielectric lenses," International Journal of Infrared and Millimeter Waves, vol. 14, no. 10, pp. 1905-1924, 1993.

[8] J. J. Lee, Handbook of Microwave and Optical Components, John Wiley \& Sons, New York, NY, USA, 1989.

[9] B. Chantraine-Barés, R. Sauleau, L. Le Coq, and K. Mahdjoubi, "A new accurate design method for millimeter-wave homogeneous dielectric substrate lens antennas of arbitrary shape," IEEE Transactions on Antennas and Propagation, vol. 53, no. 3, pp. 1069-1082, 2005.

[10] T. Dang, J. Yang, and H.-X. Zheng, "An integrated lens antenna design with irregular lens profile," in Proceedings of the 5th Global Symposium on Millimeter-Waves (GSMM '12), pp. 212215, Harbin, China, May 2012.

[11] T. Jaschke, B. Rohrdantz, and A. F. Jacob, "A flexible surface description for arbitrarily shaped dielectric lens antennas," in Proceedings of the German Microwave Conference (GeMIC '14), pp. 1-4, Aachen, Germany, March 2014.

[12] A. P. Pavacic, D. L. del Río, J. R. Mosig, and G. V. Eleftheriades, "Three-dimensional ray-tracing to model internal reflections in off-axis lens antennas," IEEE Transactions on Antennas and Propagation, vol. 54, no. 2, pp. 604-612, 2006.

[13] A. Neto, S. Maci, and P. J. I. de Maagt, "Reflections inside an elliptical dielectric lens antenna," IEE Proceedings-Microwaves, Antennas and Propagation, vol. 145, no. 3, pp. 243-247, 1998.

[14] J. Gielis, "A generic geometric transformation that unifies a wide range of natural and abstract shapes," American Journal of Botany, vol. 90, no. 3, pp. 333-338, 2003.

[15] M. Simeoni, R. Cicchetti, A. Yarovoy, and D. Caratelli, "Plasticbased supershaped dielectric resonator antennas for wide-band applications," IEEE Transactions on Antennas and Propagation, vol. 59, no. 12, pp. 4820-4825, 2011.

[16] C. A. Balanis, Antenna Theory: Analysis and Design, John Wiley and Sons, Hoboken, NJ, USA, 3rd edition, 2005.

[17] P. Bia, D. Caratelli, L. Mescia, and J. Gielis, "Electromagnetic characterization of supershaped lens antennas for highfrequency applications," in Proceedings of the 43rd European Microwave Conference (EuMC '13), pp. 1679-1682, Nuremberg, Germany, October 2013.

[18] P. Bia, D. Caratelli, L. Mescia, and J. Gielis, "Analysis and synthesis of supershaped dielectric lens antennas," IET Microwaves, Antennas \& Propagation, vol. 9, no. 14, pp. 1497-1504, 2015. 


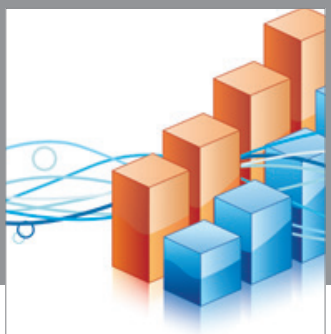

Advances in

Operations Research

vatem alat4

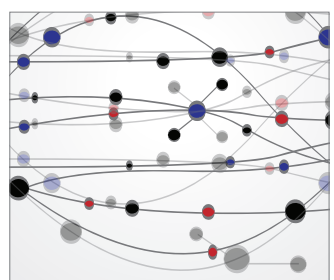

\section{The Scientific} World Journal
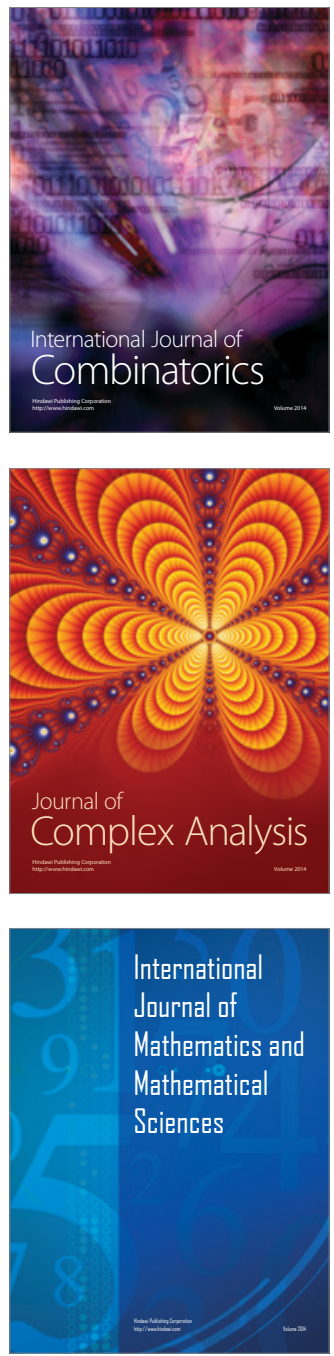
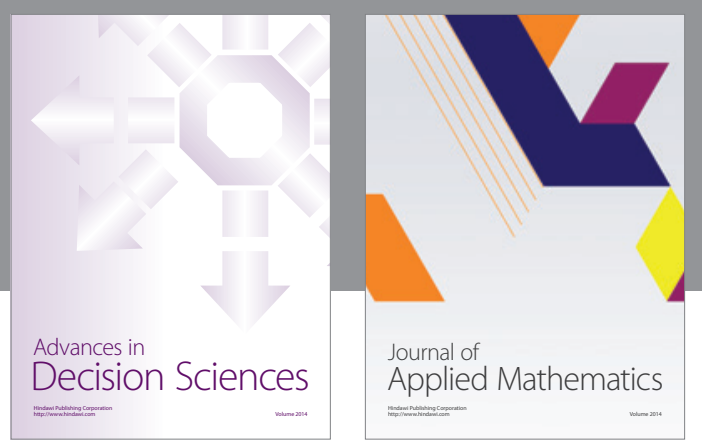

Algebra

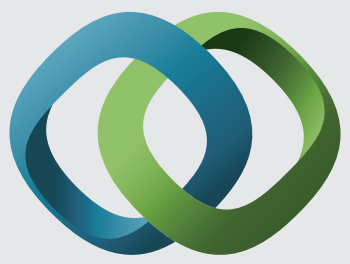

\section{Hindawi}

Submit your manuscripts at

http://www.hindawi.com
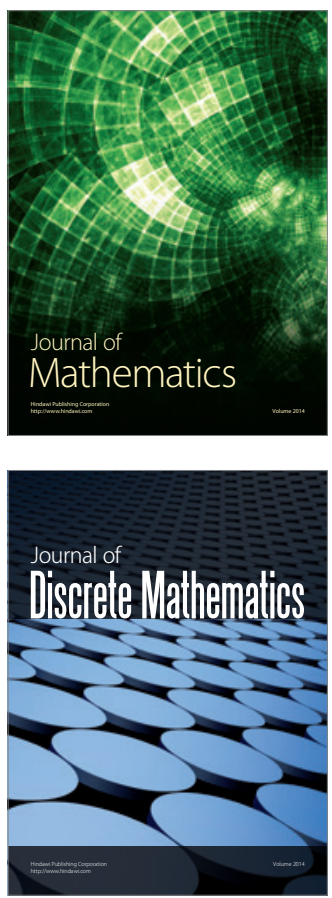

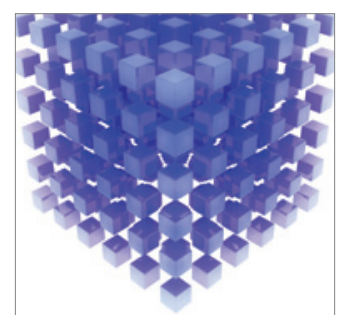

Mathematical Problems in Engineering
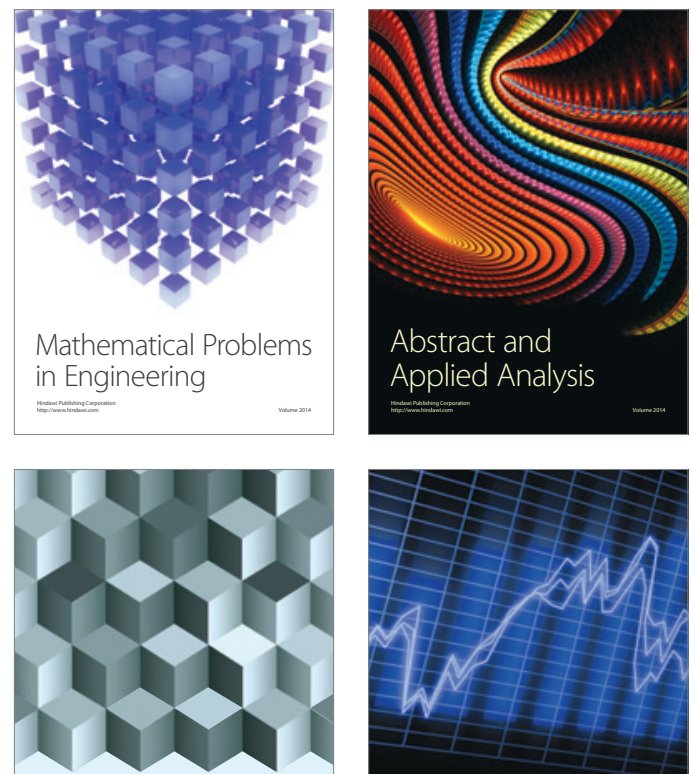

Journal of

Function Spaces

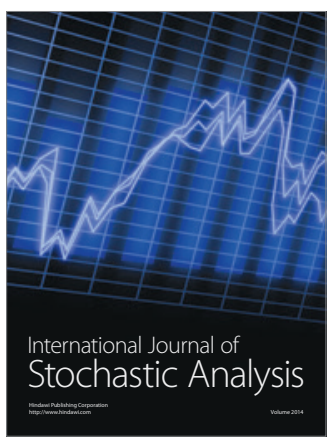

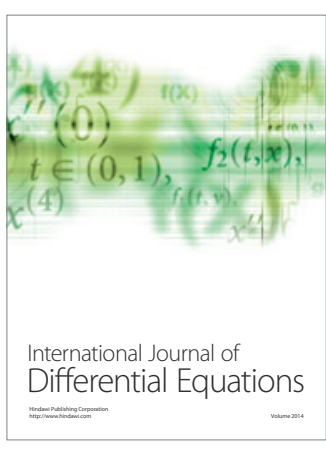
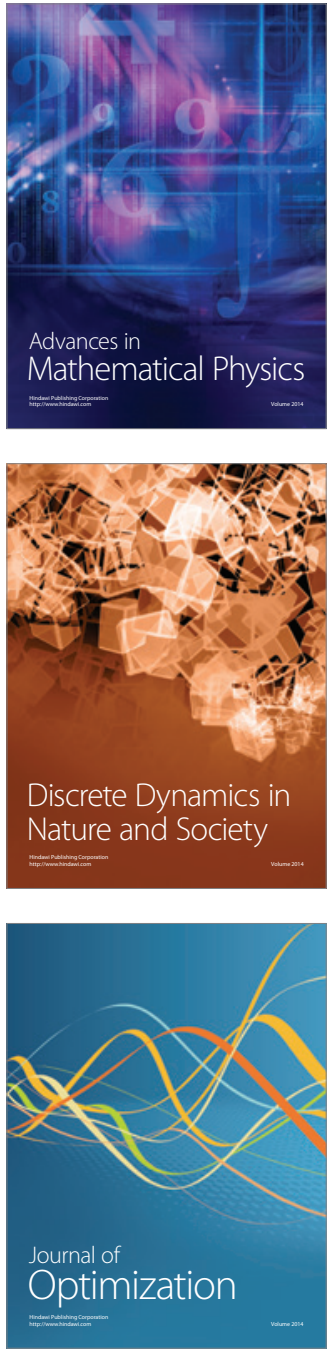